\title{
Determination of Weed Species in Kiwifruit Orchards of Ordu Province- Turkey
}

\author{
Hikmet YONAT ${ }^{1}$, Onur KOLÖREN ${ }^{2 *}$ \\ ${ }^{1}$ Ordu University, Graduate School of Natural and Applied Sciences, Ordu-Turkey \\ ${ }^{2}$ University of Ordu, Faculty of Agriculture, Department of Plant Protection, Ordu-Turkey \\ *Corresponding author: koloren@yahoo.com \& koloren@odu.edu.tr
}

\begin{abstract}
This study was conducted to determine weed species, their frequencies (\%), coverage areas (\%) and their densities (plant/ $\mathrm{m}^{2}$ ) in kiwifruit orchards of Ordu province in 2015. As a method Ordu was divided into four parts of the research area; Altınordu-Gülyalı, Ulubey-Kabadüz, Perşembe-Fatsa-Çamaş, IkizceÜnye-Çaybaşı. The study was carried out in two different periods which are April-May and SeptemberOctober in the year of 2015. Study was started from the center of Ordu and stopped for every $5 \mathrm{~km}$ to make four different examinations of randomly selected $1 \mathrm{~m}^{2}$ area of 1 da the kiwifruit orchard. During examinations weed species, their frequencies (\%), coverage area (\%) and their densities (plant $/ \mathrm{m}^{2}$ ) were determined. Eighyt six weed species belonging to 32 families were determined in the survey which was carried out in 26 kiwi orchards. At the end of this survey which is conducted in two different periods, general weed coverage is found out to be $82.27 \%$ for the first period (April-May) and $80.12 \%$ for the second period (September-October). Among these families the largest family was found to be Asteraceae having 18 species. In the first period (April-May) 71 species were identified belonging to 30 families and the most frequently encountered weed species was Convolvulus arvensis L. (field bindweed) by $69.23 \%$. In the second period (September-October) 67 species were identified belonging to 30 families and the most frequently encountered weed species was C. arvensis L. by $53.85 \%$.
\end{abstract}

Key Words: Kiwifruit, Ordu, Weed, frequency, Convolvulus arvensis L.

\section{Ordu İli Kivi Bahçelerinde Görülen Yabancı Ot Türlerinin Belirlenmesi}

Öz

Çalışma, Ordu ili kivi bahçelerinde görülen yabancı ot türlerinin, rastlama sıklıkları (\%), kaplama alanları (\%) ve yoğunlukları (bitki/m²) belirlenmesi amacı ile 2015 yılında Ordu ilinde yürütülmüştür. il dört bölgeye ayrılarak (Altınordu-Gülyalı, Ulubey-Kabadüz, Perşembe-Fatsa-Çamaş, Ikizce-Ünye-Çaybaşı) Nisan-Mayıs ve Eylül-Ekim ayları olmak üzere iki farklı dönemde, Ordu (Merkez)'dan başlamak üzere herbeş km'de bir durularak kivi bahçelerinde bir da'lık alan içerisinde dört kez bir $\mathrm{m}^{2 \prime}$ lik çerçeve atılarak çerçeve içerisinde bulunan yabancı ot türleri, rastlama sıklıkları (\%), kaplama alanları (\%) ve yoğunlukları (adet/m²) saptanmıştır. Yirmialtı kivi bahçesinde yapılan sürveylerde 32 familyaya ait 86 yabancı ot türü tespit edilmiştir. Genel yabancı otlanma (\%) birinci dönemde (Nisan-Mayıs) \% 82.27, ikinci dönemde (Eylül-Ekim) ise \% 80.12 olarak bulunmuştur. Bulunan familyalar içerisinde en geniş familya 18 tür ile Asteraceae familyası olmuştur. Birinci dönemde (Nisan-Mayıs) 30 familyaya ait 71 yabancı ot türü tespit edilmiş ve en fazla rastlanılan yabancı ot türü Convolvulus arvensis L. (Tarla sarmaşığı) \% 69.23 olmuştur. İkinci dönemde (Eylül-Ekim) ise 30 familyaya ait 67 tür tespit edilmiş ve en fazla rastlanılan yabancı ot türü yine $C$. arvensis L. \% 53.85 olmuştur.

Anahtar Kelimeler: Kivi, Ordu, Yabancı ot, rastlama sıklığı, Convolvulus arvensis L. 


\section{Introduction}

Kiwifruit (Actinidia deliciosa (Chev.) C.F.Liang \& A.R.Ferguson) is one of the least known fruit whereas it has a highly increasing production capacity in recent years. With low calorific value and reach vitamin and mineral substance content, it has a great demand (Anonymous, 2012). In terms of production volume of kiwifruit Turkey is in the rank of 7 whereas it is in the second rank in terms of production area (ha) among producer countries (Anonymous, 2013a). Turkey's convenient geological structure for the cultivation of kiwi and the increasing amount of consumption has made the kiwi production more attractive and the manufacturers shift to production of kiwi. Considering the kiwifruit production in our country Yalova is the first with 18.892 tons, Ordu is the second with 6.263 tons and Rize is the third with 5.126 tons (Anonymous, 2015). The largest area used for kiwi production in Turkey is in the Black Sea region by $70 \%$. In this region Ordu, Rize, Trabzon, Samsun and Artvin provinces have the maximum production capacity (Anonymous, 2014a).

In our country, economically important 60 cultivated plants are affected by more than 475 pests. 265 of them are pests (insects), 140 of them are pathogens and also more than 70 of them are weed species. It is not possible in conventional agriculture, organic agriculture and good agricultural practices and quality systems to get enough products without combating these harmful organisms (Tiryaki, 2011). Weeds are one of the important problems in kiwifruit orchards. Our country is struggling with plant protection problems in kiwifruit growing. Plant protection problems in the cultivation of kiwifruit in our country are performed directly for diseases and insects but indirectly for weeds. Weeds compete with kiwifruit for light, water and minerals, hence amount of yield decreases. On the other hand, weeds accommodate insects and diseases which are harmful for kiwifruit plant (Anonymous, 2012). In a study which is conducted in California University, weed control in the first four years after planting in kiwifruit orchards was determined to be very important. In the first four years after planting, kiwifruit has less chance to compete with weeds. If agricultural protection is not done for the first four years, weeds cause kiwifruits to stop growing and get dry. It is stated that only after four years kiwifruit has the necessary high to shade and so repress weeds (Anonymous, 2014b).

The purpose of this study, was to determine weed species, frequencies (\%), coverage areas (\%) and densities (plant $/ \mathrm{m}^{2}$ ) in the kiwifruit orchards in Ordu.

\section{Material and Methods}

Main material is weed species that present in 26 kiwifruit orchards in Ordu. This study was conducted in 2015 to determine weed species, their frequency (\%), coverage area (\%) and their densities (plant $\left./ \mathrm{m}^{2}\right)$ in kiwifruit orchards in Ordu. As a method Ordu was divided into 4 parts of the research area; Altınordu-Gülyalı, Ulubey-Kabadüz, Perşembe-Fatsa-Çamaş, İkizce-Ünye-Çaybaşı (Figure 1). The study was carried on in two different periods that are April-May and September-October in the year of 2015. It was started from center of Ordu and stopped for every 5 kilometers to make 4 different examination of randomly selected 1 $\mathrm{m}^{2}$ area of 1 decare in 26 kiwi orchards. This selection was done by throwing a $1 \times 1$ wooden frame onto the area randomly. 
During examination weed species, their frequencies (\%), coverage area (\%) and their densities (plant $/ \mathrm{m}^{2}$ ) are determined due to Odum (1971). A random selection was done by diagonal line the area starting from the inner part of the land to eliminate border effect. Weeds were classified according to the Flora of Turkey (Davis, 1965-1988) and Ackerunkraeuter Europas (Hanf, 1990). The Turkish names of weeds were obtained from Uluğ et al., (1993) and Güner et al., (2012).

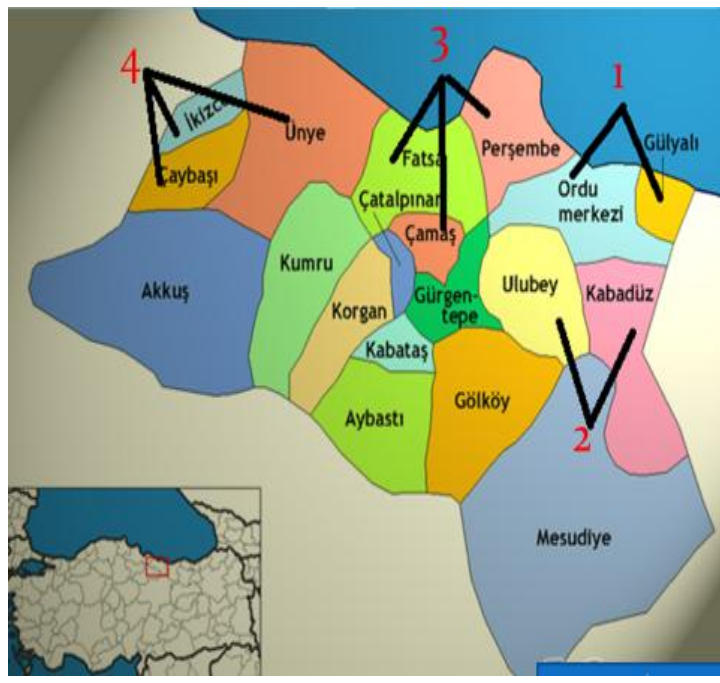

Figure 1. The map of research area (Anonymous, 2013b).

Şekil 1. Araştırma yapılan alanın haritası (Anonim, 2013b)

\section{Results and Discussion}

It has been determined that the rate of the general weed coverage (\%) of the result of a survey conducted in the Ordu 26 kiwifruit orchards were high. Compared with the general weed coverage (\%) values determined in both periods, it was determined that the highest general weed was $82.27 \%$ in the first period (April-May). In the second period (September-October) it was determined as $80.12 \%$. It shows that weed species in the first period were higher than the second period.
According to surveys performed in kiwifruit orchards of Ordu in 2015 during first period (April-May) and second period (September-October) 86 weed species belonging to 32 families were determined. In terms of the number of species Asteraceae was the first one with 18 different species, while the Poaceae family was the second family with 14 species. As for the other families, Lamiaceae had 6 species, Fabaceae had 5 species, Polygonaceae had 5 species, Apiaceae had 3 species. Amaranthaceae, Brassicaceae, Caryophyllaceae, Cyperaceae, Euphorbiaceae, Geraniaceae, Plantaginaceae, Polypodiaceae and Rosaceae had 2 species for each and the rest had one species for each weed. Among the 32 families, 2 species of Polypodiaceae family, 1 species of Equisetaceae family, and 83 species of 30 families were identified as weeds. Of these families, 28 were dicotyledonous, 2 were monocotyledonous and 2 were non-seeded. 45 of the weed species found were perennial, 38 were single year and 3 were two year respectively. In the first period (April-May) 71 species were identified belonging to 30 families, In the second period (September-October) 67 species were identified belonging to 30 families. The number of weed species determined in both periods was 52 (Table 1; Table 2).

In the first period (April-May) the first five weed species with respect to frequencies (\%) were; Convolvulus arvensis L. (field bindweed) $69.23 \%$, Artemisia vulgaris L (mugwort) $65.38 \%$, Stellaria media (L.) Vill. (chickweed) $65.38 \%$, Urtica dioica L. (stinging nettle) $61.54 \%$, Poa trivialis L. (rough stanlk bluegrass) $57.69 \%$ whereas in the second period (September-October) the first five weed species with respect to frequencies (\%) were; Convolvulus arvensis L. 
(field bindweed) $53.85 \%$, Amaranthus retroflexus L. (redroot pigweed) $50.00 \%$, Urtica dioica L. (stinging nettle) $46.15 \%$, Setaria glauca (L.) P.B. (yellow foxtail) 46.15 $\%$, Artemisia vulgaris L. (mugwort) (Table 3). In the first period (April-May) the first five weed species with respect to general coverage (\%) were; Poa trivialis L. (rough stanlk bluegrass) $10.37 \%$, Artemisia vulgaris L. (mugwort) $8.07 \%$, Bromus tectorum L. (cheatgrass) $5.48 \%$, Lolium sp. (ryegrass)
$3.67 \%$, Medicago arabica (L.) Huds. (spotted medick) $3.61 \%$ whereas in the second period (September-October) the first five weed species with respect to general coverage (\%) were; Setaria glauca (L.) (yellow foxtail) 11.37 \%, Echinochloa crus-galli (L.) P. Beauv. (barnyardgrass) $6.68 \%$, Urtica dioica L. (stinging nettle) $5.13 \%$, Artemisia vulgaris L. (mugwort) $4.68 \%$, Stellaria media (L.) Vill. (chickweed) $4.02 \%$ (Table 3).

Table 1. Distribution of weeds families in kiwifruit orchards in Ordu Çizelge 1. Ordu ili kivi bahçelerinde yabancı otların familyalarına göre dağılımı

\begin{tabular}{|c|c|c|c|}
\hline $\begin{array}{l}\text { Families } \\
\text { Familyalar }\end{array}$ & $\begin{array}{c}\text { Number of weed species } \\
\text { (1 } 1^{\text {st }} \text { Period-April/May) } \\
\text { Yabancı ot tür sayısı } \\
\text { (1. Dönem-Nisan/Mayıs) }\end{array}$ & $\begin{array}{c}\text { Number of weed species } \\
\left(2^{\text {nd }} \text { Period-September/ }\right. \\
\text { October }) \\
\text { Yabancı ot tür sayısı } \\
\text { 2. Dönem (Eylül-Ekim) }\end{array}$ & $\begin{array}{l}\text { Number of weed species } \\
\left(1^{\text {st }} \text { and } 2^{\text {nd }} \text { Period) }\right. \\
\text { Yabancı ot tür sayısı } \\
\text { (1. ve 2. Dönem) }\end{array}$ \\
\hline Amaranthaceae & 2 & 2 & 2 \\
\hline Apiaceae & 3 & 1 & 1 \\
\hline Araceae & 1 & 1 & 1 \\
\hline Asteraceae & 14 & 12 & 8 \\
\hline Boraginaceae & 1 & - & - \\
\hline Brassicaceae & 2 & 1 & 1 \\
\hline Caprifoliaceae & 1 & 1 & 1 \\
\hline Caryophyllaceae & 2 & 2 & 2 \\
\hline Commelinaceae & 1 & 1 & 1 \\
\hline Convolvulaceae & 1 & 1 & 1 \\
\hline Cyperaceae & 2 & 2 & 2 \\
\hline Equisetaceae & 1 & 1 & 1 \\
\hline Euphorbiaceae & 2 & 2 & 2 \\
\hline Fabaceae & 5 & 4 & 4 \\
\hline Geraniaceae & 2 & 2 & 2 \\
\hline Lamiaceae & 3 & 5 & 2 \\
\hline Lythraceae & - & 1 & - \\
\hline Malvaceae & 1 & 1 & 1 \\
\hline Oxalidaceae & 1 & 1 & 1 \\
\hline Phytolaccaceae & - & 1 & - \\
\hline Plantaginaceae & 2 & 2 & 2 \\
\hline Poaceae & 11 & 10 & 7 \\
\hline Polygonaceae & 2 & 5 & 2 \\
\hline Polypodiaceae & 2 & 1 & 1 \\
\hline Portulacaceae & 1 & 1 & 1 \\
\hline Primulaceae & 1 & 1 & 1 \\
\hline Ranunculaceae & 1 & - & - \\
\hline Rosaceae & 2 & 1 & 1 \\
\hline Rubiaceae & 1 & 1 & 1 \\
\hline Scrophulariaceae & 1 & 1 & 1 \\
\hline Solanaceae & 1 & 1 & 1 \\
\hline Urticaceae & 1 & 1 & 1 \\
\hline $\begin{array}{c}\text { Total } \\
\text { Toplam }\end{array}$ & 71 & 67 & 52 \\
\hline
\end{tabular}


In the first period (April-May) the first five weed species with respect to densities (plant $/ \mathrm{m}^{2}$ ) were; Poa trivialis L. (rough stanlk bluegrass) 16.27, Artemisia vulgaris L. (mugwort) 11.38, Bromus tectorum L. (cheatgrass) 7.31, Lolium sp. (ryegrass) 6.38, Medicago arabica (L.) Huds. (spotted medick) 5.08 whereas in the second period (September-October) the first five weed species with respect to densities (plant $/ \mathrm{m}^{2}$ ) were; Setaria glauca L. (yellow foxtail) 14.42, Echinochloa crus-galli (L.) P. Beauv. (barnyardgrass) 10.27, Stellaria media (L.) Vill. (chickweed) 5.62, Artemisia vulgaris L. (mugwort) 5.31, Urtica dioica L. (stinging nettle) 5.23 (Table 3).

86 weed species identified in the surveys conducted, 56 were found to be similar to the species mentioned in Deveci (2003). Important weed species showing similarity; C. arvensis A. vulgaris, E. crus galli, S. glauca, $R$. crispus, $U$. dioica, Lolium spp., A. retroflexus, C. flacca, B. perennis, S. asper, M. arabica, G. hederacea, O. acetosella, P. lanceolata, $P$. major, $D$. sanguinalis, $A$. arvensis, $S$. nigrum, $M$. annua and $T$. repens. As a result of study in kiwifruit orchards, it was found that species belonging to the families of Asteraceae and Poaceae were the most similar. The similarity rate is also high in terms of weed species. The rates of frequencies (\%), coverage areas (\%) and densities (plant $/ \mathrm{m}^{2}$ ) of similar weed species were found to vary. Some weeds detected in surveys are reported as important weed species found in Anonymous (2003), a survey of kiwifruit orchards in the state of California (USA); P. annua, E. crus-galli, S. media, S. vulgaris, $D$. sanguinalis, $C$. canadensis, $C$. album, S. arvensis, P. oleracea, C. bursapastoris, S. halepense, $P$. dilatatum, $C$. dacytlon, R. crispus, C. arvensis, T. officinale, C. esculentus, Malva sp., and A. fatua were similar to each other and these weed species were also important problems in the kiwifruit orchards in which we study.

Table 2. General coverage (\%), densities (plant $/ \mathrm{m}^{2}$ ) and frequencies (\%) of weed species at $1^{\text {st }}$ period (April-May) and $2^{\text {nd }}$ period (September-October) in kiwifruit orchards in Ordu

Çizelge 2. Ordu ili kivi bahçelerinde 1. Dönem (Nisan-Mayıs) ile 2. Dönemde (Eylül-Ekim) bulunan yabancı ot türleri ve bunların rastlama sıklıkları (\%), genel kaplama (\%) ile yoğunlukları (bitki/m²)

\begin{tabular}{|c|c|c|c|c|c|c|}
\hline \multirow[b]{2}{*}{$\begin{array}{l}\text { Weed Species } \\
\text { Yabancı ot türleri }\end{array}$} & \multicolumn{3}{|c|}{$\begin{array}{l}1^{\text {st }} \text { Period (April-May) } \\
\text { 1. } \quad \text { Dönem (Nisan-Mayıs) }\end{array}$} & \multicolumn{3}{|c|}{$\begin{array}{l}2^{\text {nd }} \text { Period (September-October) } \\
\text { 2. Dönem (Eylül-Ekim) }\end{array}$} \\
\hline & $\begin{array}{c}F \\
(\%)^{*}\end{array}$ & $\begin{array}{l}\mathrm{GC} \\
(\%)^{*}\end{array}$ & $\begin{array}{c}\mathrm{D} \\
\left.\text { (plant } / \mathrm{m}^{2}\right)^{*}\end{array}$ & $\begin{array}{c}F \\
(\%)^{*}\end{array}$ & $\begin{array}{l}\mathrm{GC} \\
(\%)^{*}\end{array}$ & $D\left(\text { plant } / \mathrm{m}^{2}\right)^{*}$ \\
\hline \multicolumn{7}{|l|}{ AMARANTHACEAE } \\
\hline Amaranthus retroflexus L. & 11.54 & 0.60 & 1.31 & 50.00 & 1.43 & 1.35 \\
\hline Chenopodium album L. & 7.69 & 0.13 & 0.15 & 7.69 & 0.10 & 0.12 \\
\hline \multicolumn{7}{|l|}{ APIACEAE } \\
\hline Aethusa cynapium L. & 23.08 & 1.12 & 1.04 & 3.85 & 0.06 & 0.04 \\
\hline Bifora radians Bieb. & 3.85 & 0.09 & 0.12 & & & \\
\hline Daucus carota $\mathrm{L}$. & 3.85 & 0.06 & 0.08 & & & \\
\hline \multicolumn{7}{|l|}{ ARACEAE } \\
\hline Arum maculatum L. & 11.54 & 0.34 & 0.62 & 7.69 & 0.27 & 0.31 \\
\hline \multicolumn{7}{|l|}{ ASTERACEAE } \\
\hline Anthemis arvensis L. & 7.69 & 0.13 & 0.15 & & & \\
\hline Arctium lappa L. & 30.77 & 0.53 & 0.62 & 30.77 & 0.42 & 0.38 \\
\hline
\end{tabular}




\begin{tabular}{|c|c|c|c|c|c|c|}
\hline Artemisia vulgaris $\mathrm{L}$. & 65.38 & 8.07 & 11.38 & 42.31 & 4.68 & 5.31 \\
\hline Bellis perennis L. & 53.85 & 2.53 & 2.92 & & & \\
\hline Cichorium intybus L. & 15.38 & 0.23 & 0.27 & 3.85 & 0.05 & 0.04 \\
\hline Cirsium arvense (L.) Scop. & 19.23 & 0.23 & 0.31 & & & \\
\hline Conyza bonariensis (L.) Cronquist. & 3.85 & 0.06 & 0.08 & 3.85 & 0.22 & 0.12 \\
\hline Conyza canadensis (L.) Cronquist. & 7.69 & 0.15 & 0.23 & 23.08 & 1.31 & 0.92 \\
\hline Eupatorium cannabinum L. & & & & 3.85 & 0.22 & 0.27 \\
\hline Lactuca serriola L. & 38.46 & 0.79 & 0.96 & & & \\
\hline Matricaria chamomilla L. & 15.38 & 0.52 & 0.58 & & & \\
\hline Pulicaria dysenterica (L.) Cass. & & & & 3.85 & 0.15 & 0.08 \\
\hline Senecio vulgaris $\mathrm{L}$. & 7.69 & 0.11 & 0.15 & & & \\
\hline Sonchus asper (L.) Hill & & & & 7.69 & 0.14 & 0.15 \\
\hline Sonchus oleraceus L. & 11.54 & 0.3 & 0.35 & 3.85 & 0.09 & 0.12 \\
\hline Taraxacum officinale F.H. Wigg & 19.23 & 0.36 & 0.42 & 7.69 & 0.43 & 0.42 \\
\hline Tragopogon sp. & 11.54 & 0.21 & 0.31 & 11.54 & 0.27 & 0.23 \\
\hline Xanthium strumarium L. & & & & 7.69 & 0.08 & 0.15 \\
\hline \multicolumn{7}{|l|}{ BORAGINACEAE } \\
\hline Anchusa azurea Miller. & 3.85 & 0.03 & 0.04 & & & \\
\hline \multicolumn{7}{|l|}{ BRASSICACEAE } \\
\hline Capsella bursa-pastoris (L.) Medik. & 19.23 & 0.38 & 0.38 & 3.85 & 0.03 & 0.04 \\
\hline Sinapis arvensis L. & 19.23 & 0.54 & 0.81 & & & \\
\hline \multicolumn{7}{|l|}{ CAPRIFOLIACEAE } \\
\hline Sambucus nigra L. & 11.54 & 0.26 & 0.27 & 3.85 & 0.27 & 0.31 \\
\hline \multicolumn{7}{|l|}{ CARYOPHYLLACEAE } \\
\hline Cerastium tomentosum L. & 26.92 & 2.40 & 3.15 & 3.85 & 0.08 & 0.12 \\
\hline Stelleria media (L.) Vill. & 65.38 & 3.40 & 4.12 & 23.08 & 4.02 & 5.62 \\
\hline \multicolumn{7}{|l|}{ COMMELINACEAE } \\
\hline Commelina communis L. & 11.54 & 0.42 & 0.54 & 15.38 & 2.05 & 1.92 \\
\hline \multicolumn{7}{|l|}{ CONVOLVULACEAE } \\
\hline Convolvulus arvensis L. & 69.23 & 2.86 & 3.27 & 53.85 & 1.87 & 1.81 \\
\hline \multicolumn{7}{|l|}{ CYPERACEAE } \\
\hline Carex flacca Schreber & 11.54 & 2.99 & 4.08 & 15.38 & 2.39 & 2.38 \\
\hline Cyperus rotundus L. & 3.85 & 0.21 & 0.31 & 3.85 & 0.33 & 0.31 \\
\hline \multicolumn{7}{|l|}{ EQUISETACEAE } \\
\hline Equisetum arvense L. & 15.38 & 0.36 & 0.58 & 3.85 & 0.19 & 0.23 \\
\hline \multicolumn{7}{|l|}{ EUPHORBIACEAE } \\
\hline Euphorbia helioscopia L. & 42.31 & 0.97 & 1.27 & 11.54 & 0.24 & 0.27 \\
\hline Mercurialis annua $\mathrm{L}$. & 11.54 & 0.31 & 0.38 & 23.08 & 2.00 & 2.04 \\
\hline \multicolumn{7}{|l|}{ FABACEAE } \\
\hline Glycyrrhiza glabra L. & 19.23 & 0.43 & 0.65 & & & \\
\hline Medicago arabica (L.) Huds. & 50.00 & 3.61 & 5.08 & 15.38 & 1.57 & 1.62 \\
\hline Medicago sativa $\mathrm{L}$. & 15.38 & 0.54 & 0.73 & 30.77 & 2.44 & 2.12 \\
\hline Trifolium repens $\mathrm{L}$. & 53.85 & 2.89 & 4.27 & 11.54 & 0.87 & 0.92 \\
\hline Vicia sativa $\mathrm{L}$. & 38.46 & 1.49 & 1.85 & 3.85 & 0.25 & 0.27 \\
\hline \multicolumn{7}{|l|}{ GERANIACEAE } \\
\hline Erodium acaule (L.) Becherer and Thell. & 3.85 & 0.45 & 0.65 & 15.38 & 0.85 & 0.85 \\
\hline Geranium dissectum $\mathrm{L}$. & 23.08 & 0.92 & 0.96 & 19.23 & 1.44 & 1.12 \\
\hline \multicolumn{7}{|l|}{ LAMIACEAE } \\
\hline Ballato nigra L. & 11.54 & 0.34 & 0.73 & & & \\
\hline Glechoma hederacea L. & 3.85 & 0.02 & 0.04 & 15.38 & 1.64 & 1.85 \\
\hline Lamium purpureum L. & & & & 3.85 & 0.12 & 0.08 \\
\hline Melissa officinalis L. & 34.62 & 1.77 & 2.00 & 19.23 & 1.44 & 1.58 \\
\hline Prunella vulgaris $\mathrm{L}$. & & & & 3.85 & 0.22 & 0.27 \\
\hline
\end{tabular}




\begin{tabular}{|c|c|c|c|c|c|c|}
\hline Salvia forskahlei L. & & & & 7.69 & 0.50 & 0.65 \\
\hline \multicolumn{7}{|l|}{ LYTHRACEAE } \\
\hline Lythrum salicaria $\mathrm{L}$. & & & & 7.69 & 0.57 & 0.73 \\
\hline \multicolumn{7}{|l|}{ MALVACEAE } \\
\hline Malva neglecta $\mathrm{L}$. & 7.69 & 0.09 & 0.12 & 11.54 & 1.00 & 1.12 \\
\hline \multicolumn{7}{|l|}{ OXALIDACEAE } \\
\hline Oxalis acetosella $\mathrm{L}$. & 11.54 & 0.58 & 0.81 & 3.85 & 0.18 & 0.12 \\
\hline \multicolumn{7}{|l|}{ PHYTOLACCACEAE } \\
\hline Phytolacca americana L. & & & & 7.69 & 0.13 & 0.08 \\
\hline \multicolumn{7}{|l|}{ PLANTAGINACEAE } \\
\hline Plantago lanceolata L. & 11.54 & 0.39 & 0.35 & 3.85 & 0.15 & 0.12 \\
\hline Plantago major L. & 11.54 & 0.27 & 0.27 & 19.23 & 0.74 & 0.88 \\
\hline \multicolumn{7}{|l|}{ POACEAE } \\
\hline Agropyrum repens L. & 23.08 & 1.32 & 3.00 & 7.69 & 0.99 & 0.73 \\
\hline Alopecurus myosuroides Hudson & 7.69 & 0.39 & 0.54 & 7.69 & 0.69 & 0.54 \\
\hline Avena fatua $\mathrm{L}$. & 19.23 & 1.26 & 1.27 & & & \\
\hline Avena sativa $\mathrm{L}$. & & & & 3.85 & 0.17 & 0.19 \\
\hline Bromus tectorum $\mathrm{L}$. & 57.69 & 5.48 & 7.31 & 7.69 & 0.99 & 1.12 \\
\hline Cynodon dactylon (L.) Pers. & 11.54 & 1.62 & 1.62 & & & \\
\hline Digitaria sanguinalis (L.) Scop. & & & & 19,23 & 2,49 & 3,12 \\
\hline Echinochloa crus galli (L.) P.Beauv. & 15.38 & 1.08 & 1.73 & 34.62 & 6.68 & 10.27 \\
\hline Lolium spp. & 42.31 & 3.67 & 6.38 & 30.77 & 3.04 & 3.42 \\
\hline Oplismenus undulafolius (Ard.) P. Beauv. & 7.69 & 0.11 & 0.15 & 38.46 & 3.92 & 4.19 \\
\hline Paspalum dilatatum Poiret & & & & 7.69 & 0.30 & 0.46 \\
\hline Poa trivialis $\mathrm{L}$. & 57.69 & 10.37 & 16.27 & & & \\
\hline Setaria glauca (L.) P.Beauv. & 23.08 & 3.15 & 4.62 & 46.15 & 11.37 & 14.42 \\
\hline Sorghum halepense (L.) Pers. & 11.54 & 0.80 & 0.81 & & & \\
\hline \multicolumn{7}{|l|}{ POLYGONACEAE } \\
\hline Polygonum aviculare L. & 3.85 & 0.21 & 0.15 & 3.85 & 1.09 & 0.65 \\
\hline Polygonum hydropiper L. & & & & 19.23 & 2.79 & 2.46 \\
\hline Polygonum lapathifolium L. & & & & 11.54 & 0.28 & 0.42 \\
\hline Polygonum persicaria L. & & & & 3.85 & 0.29 & 0.42 \\
\hline Rumex crispus L. & 46.15 & 1.70 & 2.15 & 30.77 & 0.59 & 0.73 \\
\hline \multicolumn{7}{|l|}{ POLYPODIACEAE } \\
\hline Dryopteris filix-max (L.) Schott & 11.54 & 0.44 & 0.35 & 15.38 & 0.69 & 0.69 \\
\hline Pteridium aquilinum (L.) Kuhn & 3.85 & 0.10 & 0.15 & & & \\
\hline \multicolumn{7}{|l|}{ PORTULACACEAE } \\
\hline Portulaca olaracea L. & 3.85 & 0.04 & 0.04 & 3.85 & 0.09 & 0.12 \\
\hline \multicolumn{7}{|l|}{ PRIMULACEAE } \\
\hline Anagallis arvensis L. & 11.54 & 0.16 & 0.19 & 3.85 & 0.20 & 0.19 \\
\hline \multicolumn{7}{|l|}{ RANUNCULACEAE } \\
\hline Ranunculus acris $\mathrm{L}$. & 26.92 & 1.01 & 1.27 & & & \\
\hline \multicolumn{7}{|l|}{ ROSACEAE } \\
\hline Fragaria vesca L. & 3.85 & 0.04 & 0.08 & & & \\
\hline Rubus sp. & 11.54 & 0.19 & 0.08 & 3.85 & 0.03 & 0.04 \\
\hline \multicolumn{7}{|l|}{ RUBIACEAE } \\
\hline Galium aparine L. & 7.69 & 0.13 & 0.19 & 3.85 & 0.12 & 0.08 \\
\hline \multicolumn{7}{|l|}{ SCROPHULARIACEAE } \\
\hline Veronica sp. & 15.38 & 0.18 & 0.23 & 7.69 & 0.36 & 0.42 \\
\hline \multicolumn{7}{|l|}{ SOLANACEAE } \\
\hline Solanum nigrum L. & 7.69 & 0.08 & 0.08 & 19.23 & 0.32 & 0.38 \\
\hline \multicolumn{7}{|l|}{ URTICACEAE } \\
\hline Urtica dioica $\mathrm{L}$. & 61.54 & 3.26 & 3.92 & 46.15 & 5.13 & 5.23 \\
\hline
\end{tabular}

${ }^{*} \mathrm{~F}=$ Frequencies, $\mathrm{GC}=$ General Coverage, $\mathrm{D}=$ Densities 
Table 3. The most frequently observed five weeds' general coverage (\%), densities (plant $/ \mathrm{m}^{2}$ ) and frequencies (\%) in kiwifruit orchards in Ordu

Çizelge 3. Ordu ili kivi bahçelerinde en fazla görülen beş yabancı ot türünün genel kaplama (\%), yoğunluk (bitki/ $\mathrm{m}^{2}$ ) ve rastlama sıklıkları (\%)

\begin{tabular}{|c|c|c|c|c|c|c|c|}
\hline \multirow[b]{2}{*}{$\begin{array}{l}\text { Weed species } \\
\text { Yabancı ot türleri }\end{array}$} & \multicolumn{3}{|c|}{$\begin{array}{l}1^{\text {st }} \text { Period (April-May) } \\
\text { 1. Dönem (Nisan-Mayıs) }\end{array}$} & \multirow[b]{2}{*}{$\begin{array}{l}\text { Weed species } \\
\text { Yabancı ot türleri }\end{array}$} & \multicolumn{3}{|c|}{$\begin{array}{c}2^{\text {nd }} \text { Period } \\
\text { (September-October) } \\
\text { 2.Dönem (Eylül-Ekim) }\end{array}$} \\
\hline & $\begin{array}{c}F \\
(\%)^{*}\end{array}$ & $\begin{array}{c}\mathrm{GC} \\
(\%)^{*}\end{array}$ & $\begin{array}{c}\mathrm{D} \\
\left(\text { plant } / \mathrm{m}^{2}\right)^{*}\end{array}$ & & $\begin{array}{c}\mathrm{F} \\
(\%)^{*}\end{array}$ & $\begin{array}{c}\mathrm{GC} \\
(\%)^{*}\end{array}$ & $\frac{D}{\left.\text { plant } / m^{2}\right)^{*}}$ \\
\hline Convolvulus arvensis $L$. & 69.23 & 2.86 & 3.27 & Convolvulus arvensis L. & 53.85 & 1.87 & 1.81 \\
\hline Artemisia vulgaris $L$. & 65.38 & 8.07 & 11.38 & Amaranthus retroflexus $\mathrm{L}$. & 50.00 & 1.43 & 1.35 \\
\hline Stellaria media (L.) Vill. & 65.38 & 3.40 & 4.12 & Urtica dioica $\mathrm{L}$. & 46.15 & 5.13 & 5.23 \\
\hline Urtica dioica $\mathrm{L}$. & 61.54 & 3.26 & 3.92 & Setaria glauca (L.) P.B. & 46.15 & 11.37 & 14.42 \\
\hline Poa trivialis $\mathrm{L}$. & 57.69 & 10.37 & 16.27 & Artemisia vulgaris L. & 42.31 & 4.68 & 5.31 \\
\hline
\end{tabular}

${ }^{*} \mathrm{~F}=$ Frequencies, $\mathrm{GCA}=$ General Coverage, $\mathrm{D}=$ Densities

\section{Conclusions}

Even though kiwifruit farming is a new field in Turkey, production and consumption are increasing day by day. Kiwifruit farming in Turkey is mostly done especially in the Black Sea Region. Current developments show that kiwifruit can produce a variety of products in places where hazelnut and tea production are made.

Despite the rapidly increasing human population in the world, agricultural production areas are gradually decreasing. In order for humans not to suffer from food shortage, it is necessary to obtain more yield from agricultural production areas. For this, it is necessary to combat diseases, insects and weeds which damage culture plants in a correct and effective way. As a result, we able to protect our nature and we also able to produce quality and high yield.

With this study;

It has been determined that the general weed coverage (\%) is higher in the kiwifruit orchards that are surveyed.
It was determined that the population of weed was high because the amount of rainfall in Ordu province Black Sea Region is higher than other regions.

In order to obtain better yields in the kiwi production areas in our country, the plant protection method (disease agents, insects and weeds) will be reached with the right and timely struggle with the desired aim.

In this study, we have determined frequency (\%), coverage area (\%), density (plant $/ \mathrm{m}^{2}$ ) of kiwifruit orchards, which is one of the important agricultural products grown in Ordu and its districts. This work will help raise awareness of the kiwi producer and shed light on other work.

\section{Acknowledgment}

This study was supported by Ordu University Scientific Researchs Project Coordination Unit (ODU BAP, Project no: TF1458). This study was also accepted master thesis in Department of Plant Protection, Institute of Science, University of Ordu. 


\section{References}

Anonymous, 2003. A Pest Management Strategic Plan for Kiwifruit Production in CaliforniaThe California Kiwifruit Commission. http://www.ipmcenters.org/pmsp/pdf/CA KIWIFRUIT.PDF-(Date of access: 27.06.2014).

Anonymous, 2012. Kivi aşırı soğuk ve sıcağı sevmiyor. Hasad Bitkisel Üretim Aylık Tarım Dergisi, 28(331): 62-74.

Anonymous,

2013a. http://faostat3.fao.org/download/Q/QC/E - (Date of access: 27.01.2016)

Anonymous, 2013b. Ordu ismi nereden geliyor? Ordu şehir ismi hikayesi efsanesi. http://www.bilginasil.com/ordu-isminereden-geliyor-ordu-sehir-ismi-hikayesiefsanesi.html-( Date of access: 30.01.2016).

Anonymous, 2014a. Arazi Kullanım Kabiliyet Sınıfları. Ordu İli Tarım Master Planı. Gıda Tarım ve Hayvancılık Bakanlığı, Yayın no: 22, Bağcılar/İstanbul, 468s.

Anonymous, 2014b. Kiwifruit Pest Management Guidelines. Integrated Weed Management. http://www.ipm.ucdavis.edu/PMG/r43070 0111.html (Date of access: 01.07.2014).

Anonymous, 2015. http://rapory.tuik.gov.tr/1902-2016-21:03:099281100645772351291168834024.html?(Date of access:19.02.2016).

Davis, P. H., 1965-1988. Flora of Turkey and the East Aegean Islands. Edinburgh at the University Press, Edingburg, Volume 1-10.

Deveci, M., 2003. Ordu İli Kivi Bahçelerinde Bulunan Bitkiler, Bunların Yoğunlukları ve Rastlama Sıklıklarının Belirlenmesi. Karadeniz Teknik Üniversitesi Ordu Ziraat Fakültesi Ulusal Kivi ve Üzümsü Meyveler Sempozyumu, 23-25 Ekim 2003, Ordu, 197-202 s.

Güner, A., Arslan, S., Ekim, T., Vural M., Babaç, M.T., 2012. Türkiye Bitkiler Listesi (Damarlı Bitkiler). Nezahat Gökyiğit Botanik Bahçesi ve Flora Araştırmaları Derneği Yayını. İstanbul.
Hanf, M., 1990. Ackerunkraeuter Europas. Mit Ihren Keimlingen und Samen, Germany, 496 pp.

Odum, E.P., 1971. Fundamentals of Ecology. W. B. Saunders Company, Philadelphia, London, Toronto, 574 pp.

Tiryaki, O., 2011. Pestisit Kalıntı Analizlerinde Kalite Kontrol (QC) ve Kalite Güvencesi (QA). Nobel Yayın No:1635, Ankara.

Uluğ, E., Kadıoğlu, İ., Üremiş, ì., 1993. Türkiye'nin Yabancı Otları ve Bazı Özellikleri. T.C. Tarım ve Köyişleri Bakanlığı, Zirai Mücadele Araştırma Enstitüsü Müdürlüğü, No:78, Adana, 513 s. 